\title{
SUM THE MULTIPLICATIVE ODDS TO ONE AND STOP
}

\author{
MITSUSHI TAMAKI,* Aichi University
}

\begin{abstract}
We consider the optimal stopping problem of maximizing the probability of stopping on any of the last $m$ successes of a sequence of independent Bernoulli trials of length $n$, where $m$ and $n$ are predetermined integers such that $1 \leq m<n$. The optimal stopping rule of this problem has a nice interpretation, that is, it stops on the first success for which the sum of the $m$-fold multiplicative odds of success for the future trials is less than or equal to 1. This result can be viewed as a generalization of Bruss' (2000) odds theorem. Application will be made to the secretary problem. For more generality, we extend the problem in several directions in the same manner that Ferguson (2008) used to extend the odds theorem. We apply this extended result to the full-information analogue of the secretary problem, and derive the optimal stopping rule and the probability of win explicitly. The asymptotic results, as $n$ tends to $\infty$, are also obtained via the planar Poisson process approach.
\end{abstract}

Keywords: Optimal stopping; secretary problem; planar Poisson process; odds theorem; nonhomogeneous Poisson process; monotone problem; one-stage look-ahead rule

2010 Mathematics Subject Classification: Primary 60G40

\section{Introduction}

Let $n$ be a given positive integer, and suppose that $n$ independent Bernoulli trials are performed one at a time, each of which results in a success or a failure. That is, if we let $X_{j}$ equal 1 if the $j$ th trial is a success and 0 if it is a failure, then $X_{1}, X_{2}, \ldots, X_{n}$ are independent Bernoulli random variables that are observed sequentially. When we seek an optimal stopping rule of this sequential observation problem with the objective of maximizing the probability of stopping on the last success, Bruss (2000) gave an elegant solution, given below, where $p_{j}=\mathrm{P}\left\{X_{j}=1\right\}, q_{j}=1-p_{j}$, and $r_{j}=p_{j} / q_{j}$ represents the odds of success on the $j$ th trial (if $p_{j}=1$ and $r_{j}$ is taken to be $\infty$ ).

Theorem 1.1. (Bruss' sum-the-odds theorem.) For the above stopping problem, the optimal rule stops on the first success $X_{k}=1$ with $k \geq s$, if any, where

$$
s=\min \left\{k \geq 1: \sum_{j=k+1}^{n} r_{j} \leq 1\right\} .
$$

Moreover, the maximal win (i.e. achieving the objective) probability is

$$
v=\left(\prod_{j=s}^{n} q_{j}\right)\left(\sum_{j=s}^{n} r_{j}\right) .
$$

Received 2 March 2010; revision received 29 April 2010.

* Postal address: Department of Business Administration, Aichi University, Nagoya Campus, 370 Shimizu, Kurozasa, Miyoshi, Aichi 470-0296, Japan. Email address: tamaki@vega.aichi-u.ac.jp 
The optimal rule (1.1) has a nice interpretation, i.e. it stops on the first success for which the sum of the odds of success for the future trials is less than or equal to 1 (we are indifferent between stopping and continuing if the sum of the odds is equal to 1). We refer to this result as the sum-the-odds theorem (called simply STOT) according to Ferguson (2008). Bruss (2003) later showed that the best possible lower bound on the maximal probability is $\mathrm{e}^{-1}$ under some condition, more precisely, $v>\mathrm{e}^{-1}$ if $\sum_{j=1}^{n} r_{j} \geq 1$. See also Hill and Krengel (1992) and Hsiau and Yang (2000) for related works.

Bruss (2000) started his argument by raising the question of correctly guessing the last ' 6 ' when a fair die is tossed a fixed number, $n$, of times. The STOT answers this question immediately. But what about the question of correctly guessing any one of the last two ' 6 ', i.e. guessing either the last ' 6 ' or the second to last ' 6 '. This question seems a natural extension of the Bruss question, but the STOT cannot answer this question because the optimality criterion is different. In this paper we attempt to answer problems of this kind. The criterion we adopt here is more generally described as maximizing the probability of stopping on any of the last $m$ successes for a predetermined $m$ (we assume that $n>m$ unless otherwise specified, because, for $n \leq m$, the optimal rule evidently stops on the first success). The optimal rule of this problem also has a nice interpretation. That is, it can be shown that the optimal rule stops on the first success for which the sum of the $m$-fold multiplicative odds of success for the future trials is less than or equal to 1 , if we define the sum of the $j$-fold multiplicative odds of successes on the $k$ th trial by

$$
R_{k, j}=\sum_{k \leq i_{1}<i_{2}<\cdots<i_{j} \leq n} r_{i_{1}} r_{i_{2}} \cdots r_{i_{j}}
$$

for $1 \leq j \leq n-k+1$ (if $\max _{k \leq i \leq n} p_{i}=1, R_{k, j}$ is taken to be $\infty$ ) and $R_{k, j}=0$ for $j>n-k+1$. More explicitly, we have the following result.

Theorem 1.2. (Sum-the-multiplicative-odds theorem (STMOT).) For the stopping problem of maximizing the probability of stopping on any of the last $m$ successes in $n$ independent Bernoulli trials, the optimal rule stops on the first success $X_{k}=1$ with $k \geq s_{m}$, if any, where

$$
s_{m}=\min \left\{k \geq 1: R_{k+1, m} \leq 1\right\} .
$$

Moreover, the maximal win probability is

$$
v_{m}=\left(\prod_{j=s_{m}}^{n} q_{j}\right)\left(\sum_{j=1}^{m} R_{s_{m}, j}\right) .
$$

Since $R_{k+1, m}=0$ for $k \geq n-(m-1)$, we find that $s_{m} \leq n-(m-1)$ from (1.2), which implies that, as is intuitively clear, the optimal rule stops on the success if the remaining number of trials is less than $m$. Obviously, the optimal rule may be described as

$$
N_{m}^{*}=\min \left\{k \geq s_{m}: X_{k}=1\right\} .
$$

The proof of Theorem 1.2 (the STMOT) will be given in more generality in Section 2. The STMOT with $m=1$ is just the STOT. Recall the question of correctly guessing any of the last two ' 6 ' in the dice game. This problem occurs if $m=2$ and $q_{j}=q=\frac{5}{6}, r_{j}=r=\frac{1}{5}$ for each $j$ in the STMOT, and it is easy to see that $s_{2}=n-7$ if $n \geq 8$ from (1.2), because $R_{k+1,2}=\left(\begin{array}{c}n-k \\ 2\end{array}\right) r^{2} \leq 1$ is equivalent to $n-k \leq 7$. In other words, it is optimal to announce 
the first ' 6 ', if any, in the last eight tosses as being either one of the last two ' 6 '. Moreover, the probability of a correct guess is

$$
q^{8}\left[\left(\begin{array}{l}
8 \\
1
\end{array}\right) r+\left(\begin{array}{l}
8 \\
2
\end{array}\right) r^{2}\right] \approx 0.6326
$$

from (1.3).

The STOT has been extended into other directions in Ferguson (2008). We show in Section 2 that Ferguson's extension can be also made to the STMOT. In Section 3 we apply the STMOT to the celebrated secretary problem which corresponds to the special case $p_{i}=1 / i, 1 \leq i \leq n$, and examine the asymptotic behavior as $n \rightarrow \infty$. In Section 4 we consider the full-information analogue of the secretary problem, and derive the optimal rule and the corresponding probability of win explicitly. We also obtain the asymptotic results using the planar Poisson process model. See Bruss and Paindaveine (2000), Bruss and Louchard (2009), and Hsiau and Yang (2002) for other generalizations of the STOT.

\section{The general model}

In the STMOT, Bernoulli random variables $X_{1}, X_{2}, \ldots, X_{n}$ are assumed to be independent for a finite $n$ and the payoff for not stopping is assumed to be 0 . In this section we attempt to extend the STMOT into the following directions. First, an infinite number of Bernoulli trials is allowed. Second, the payoff for not stopping is $\omega$, which may be different from 0 . Third, the Bernoulli random variables are allowed to be dependent. Fourth, at stage $i$, in addition to observing $X_{i}$, other dependent random variables are allowed to be observed that may influence the assessment of the probability of success at future stages. The method we use here is to modify the original problem into a monotone stopping problem by not allowing stopping on a failure, and then apply a simple result that gives conditions for the one-stage look-ahead rule (the 1-sla rule) to be optimal in a monotone problem (see, e.g. Ferguson (2006, Chapter 5) or Chow et al. (1971)). This method is exactly the same as that which Ferguson (2008, Section 2) used to extend the STOT, so we mimic his argument throughout this section.

Since stopping on a failure is forbidden, the notion of a 'stage' must be changed accordingly, that is, a stage is defined to contain all the observations up to and including the next success if any. Let $Z_{i}, i=1,2, \ldots$, denote the set of random variables observed after success $i-1$ up to and including success $i$. If there are less than $i$ successes, we let $Z_{i}=0$, where ' 0 ' represents a special absorbing state. Thus, we treat the following general model. Let $Z_{1}, Z_{2}, \ldots$ be a stochastic process on an arbitrary space with an absorbing state called 0 . We make the assumption that, with probability 1 , the process will eventually be absorbed at 0 . We observe the process sequentially and must predict within $m$ stages in advance when the state 0 will first be hit. If we predict correctly, we win 1 , if we predict incorrectly, we win nothing, and if the process hits 0 before we predict, we win $\omega$ (it is assumed here that $\omega<1$, because, if $\omega \geq 1$, it is clearly optimal never to stop). This is a stopping rule problem in which stopping at stage $k$ yields the payoff

$$
\begin{aligned}
Y_{k} & =\omega \mathbf{1}\left(Z_{k}=0\right)+\mathbf{1}\left(Z_{k} \neq 0\right) \mathrm{P}\left\{Z_{k+m}=0 \mid g_{k}\right\}, \quad k=1,2, \ldots \\
Y_{\infty} & =\omega
\end{aligned}
$$

where $g_{k}=\sigma\left(Z_{1}, \ldots, Z_{k}\right)$ is the $\sigma$-field generated by $Z_{1}, \ldots, Z_{k}$ and $\mathbf{1}(E)$ represents the indicator function of an event $E$. The assignment $Y_{\infty}=\omega$ means that if we never stop, we 
win $\omega$. From (2.1) we evaluate

$$
\mathrm{E}\left[Y_{k+1} \mid g_{k}\right]=\omega \mathrm{P}\left\{Z_{k+1}=0 \mid g_{k}\right\}+\mathrm{P}\left\{Z_{k+1} \neq 0, Z_{k+m+1}=0 \mid g_{k}\right\} .
$$

The 1-sla rule calls for stopping at stage $k$ if $Y_{k} \geq \mathrm{E}\left[Y_{k+1} \mid g_{k}\right]$. On the set $\left\{Z_{k}=0\right\}$, this reduces to $\omega \geq \omega$, which is always true. On the set $\left\{Z_{k} \neq 0\right\}$, this reduces to

$$
\mathrm{P}\left\{Z_{k+m}=0 \mid g_{k}\right\} \geq \omega \mathrm{P}\left\{Z_{k+1}=0 \mid g_{k}\right\}+\mathrm{P}\left\{Z_{k+1} \neq 0, Z_{k+m+1}=0 \mid g_{k}\right\} .
$$

However, an absorbing state 0 implies that $\left\{Z_{j}=0\right\} \subset\left\{Z_{j+1}=0\right\}$ for each $j$, so we easily see that

$$
\mathrm{P}\left\{Z_{k+m}=0 \mid g_{k}\right\}=\mathrm{P}\left\{Z_{k+1}=0 \mid g_{k}\right\}+\mathrm{P}\left\{Z_{k+1} \neq 0, Z_{k+m}=0 \mid g_{k}\right\}
$$

and

$$
\begin{aligned}
\mathrm{P}\left\{Z_{k+1} \neq 0, Z_{k+m+1}=0 \mid g_{k}\right\}= & \mathrm{P}\left\{Z_{k+1} \neq 0, Z_{k+m}=0 \mid g_{k}\right\} \\
& +\mathrm{P}\left\{Z_{k+m} \neq 0, Z_{k+m+1}=0 \mid g_{k}\right\} .
\end{aligned}
$$

Applying these two expressions to (2.2) yields

$$
(1-\omega) \mathrm{P}\left\{Z_{k+1}=0 \mid g_{k}\right\} \geq \mathrm{P}\left\{Z_{k+m} \neq 0, Z_{k+m+1}=0 \mid g_{k}\right\} .
$$

Therefore, the 1-sla rule is

$$
N_{m}=\min \left\{k: Z_{k}=0 \text { or }\left(Z_{k} \neq 0 \text { and } \frac{W_{k}}{V_{k}} \leq 1-\omega\right)\right\} \text {, }
$$

where

$$
V_{k}=\mathrm{P}\left\{Z_{k+1}=0 \mid g_{k}\right\}, \quad W_{k}=\mathrm{P}\left\{Z_{k+m} \neq 0, Z_{k+m+1}=0 \mid g_{k}\right\} .
$$

If $V_{k}=0$ on $\left\{Z_{k} \neq 0\right\}$ then it is a mistake to stop at $k$ since we can do at least as well by continuing one more step. Therefore, in this and subsequent formulae, we take the ratio in (2.3) to be $+\infty$ when $V_{k}=0$, even if the numerator is 0 as well.

The problem is said to be monotone if when the 1-sla rule calls for stopping at any stage, then it will continue to call for stopping at all future stages no matter what the future observations turn out to be. More specifically, the problem is monotone if

$$
A_{1} \subset A_{2} \subset A_{3} \subset \cdots \quad \text { almost surely (a.s.), }
$$

where $A_{k}=\left\{Y_{k} \geq \mathrm{E}\left[Y_{k+1} \mid g_{k}\right]\right\}$ for all $k$. From this we see that a sufficient condition for the problem to be monotone is

$$
W_{k} / V_{k} \text { is a.s. nonincreasing in } k .
$$

We have the following result.

Theorem 2.1. Suppose that the process $Z_{1}, Z_{2}, \ldots$ has an absorbing state 0 such that

$$
\mathrm{P}\left\{Z_{k} \text { is absorbed at } 0\right\}=1
$$

and that the stopping problem with reward sequence (2.1) satisfies condition (2.4). Then the 1-sla rule (2.3) is optimal.

Proof. In the present context, it is obvious that $\left|Y_{n}\right|$ is bounded by $1+|\omega|$ and

$$
\lim _{n \rightarrow \infty} Y_{n}=Y_{\infty}=\omega \text {. }
$$

These are sufficient conditions under which the 1-sla rule is optimal (see Theorem 1 of Ferguson (2008)). 


\subsection{Application to the STMOT}

We return to the problem of stopping on any of the last $m$ successes of a finite sequence of possibly dependent Bernoulli trials, $X_{1}, X_{2}, \ldots, X_{n}$. We model the information given to the decision maker through an increasing sequence of $\sigma$-fields, $\mathcal{F}_{1}, \mathcal{F}_{2}, \ldots, \mathcal{F}_{n}$, and allow him/her to use a stopping rule adapted to this sequence. We assume that, for every $j$, the event $\left\{X_{j}=1\right\}$ is in $\mathcal{F}_{j}$. In the general model formulation, if the $k$ th success occurs at stage $j$ then $g_{k}=\mathcal{F}_{j}$. Assume that $\omega=0$. Then the following corollary is immediate from Theorem 2.1.

Corollary 2.1. Suppose that $n$ Bernoulli random variables $X_{1}, X_{2}, \ldots, X_{n}$ are observed sequentially. Let $\mathcal{F}_{1}, \mathcal{F}_{2}, \ldots, \mathcal{F}_{n}$ be an increasing sequence of $\sigma$-fields such that $\left\{X_{j}=1\right\}$ is in $\mathcal{F}_{j}$ for all $1 \leq j \leq n$. Let

$$
\begin{aligned}
V_{k} & =\mathrm{P}\left\{X_{k+1}+\cdots+X_{n}=0 \mid \mathscr{F}_{k}\right\}, \\
W_{k} & =\mathrm{P}\left\{X_{k+1}+\cdots+X_{n}=m \mid \mathcal{F}_{k}\right\} .
\end{aligned}
$$

Then the optimal rule is described as

$$
N_{m}=\min \left\{k \geq 1: X_{k}=1 \text { and } \frac{W_{k}}{V_{k}} \leq 1\right\},
$$

provided that the following condition is satisfied:

$$
W_{k} / V_{k} \text { is a.s. nonincreasing in } k \text {. }
$$

This may be considered as a generalized STMOT in the sense that the ratio $W_{k} / V_{k}$ in (2.7) can be written as, from (2.5) and (2.6),

$$
\sum_{k+1 \leq i_{1}<i_{2}<\cdots<i_{m} \leq n}\left(\prod_{j=1}^{m} \frac{P_{k}\left(i_{j} \mid i_{1}, i_{2}, \ldots, i_{j-1}\right)}{1-P_{k}\left(i_{j} \mid i_{1}, i_{2}, \ldots, i_{j-1}\right)}\right),
$$

if we define

$$
P_{k}\left(i_{j} \mid i_{1}, i_{2}, \ldots, i_{j-1}\right)=\mathrm{P}\left\{X_{i_{j}}=1 \mid \mathcal{F}_{k}, K\left(i_{1}, i_{2}, \ldots, i_{j}\right)\right\},
$$

where $K\left(i_{1}, i_{2}, \ldots, i_{j}\right)$ represents, on the index set $S=\{k+1, k+2, \ldots, n\}$, an information pattern described as

$$
K\left(i_{1}, i_{2}, \ldots, i_{j}\right)=\left\{X_{i}=1 \text { for } i \in\left\{i_{1}, i_{2}, \ldots, i_{j-1}\right\}, X_{i}=0 \text { for } i \in S-\left\{i_{1}, i_{2}, \ldots, i_{j}\right\}\right\}
$$

It is easy to see that Corollary 2.1 implies the STMOT. In the STMOT, the $X_{j}$ are independent and $\mathcal{F}_{j}=\sigma\left(X_{1}, \ldots, X_{j}\right)$. So the conditioning in the definition of $P_{k}\left(i_{j} \mid i_{1}, i_{2}, \ldots, i_{j-1}\right)$ may be ignored, and $P_{k}\left(i_{j} \mid i_{1}, i_{2}, \ldots, i_{j-1}\right)=p_{i_{j}}$. Then from (2.9) we have

$$
\frac{W_{k}}{V_{k}}=\sum_{k+1 \leq i_{1}<i_{2}<\cdots<i_{m} \leq n}\left(\prod_{j=1}^{m} \frac{p_{i_{j}}}{q_{i_{j}}}\right),
$$

so that in this case, $N_{m}=N_{m}^{*}$ of (1.4). We see from this that the $W_{k} / V_{k}$ are nonrandom and nonincreasing in $k$ because increasing $k$ decreases the possible number of products on the 
right-hand side of (2.10), so that (2.8) is satisfied. Thus, the problem is monotone and the 1-sla rule, $N_{m}^{*}$, is optimal. Since $v_{m}$ is expressed as

$$
v_{m}=\sum_{j=1}^{m} \mathrm{P}\left\{\sum_{i \in S^{*}} X_{i}=j\right\},
$$

where $S^{*}=\left\{s_{m}, s_{m}+1, \ldots, n\right\}$ and the $X_{i}$ are independent, we can write

$$
\begin{aligned}
\mathrm{P}\left\{\sum_{i \in S^{*}} X_{i}=j\right\} & =\sum_{s_{m} \leq i_{1}<\cdots<i_{j} \leq n}\left(\prod_{i \in\left\{i_{1}, \ldots, i_{j}\right\}} \mathrm{P}\left\{X_{i}=1\right\} \prod_{i \in S^{*}-\left\{i_{1}, \ldots, i_{j}\right\}} \mathrm{P}\left\{X_{i}=0\right\}\right) \\
& =\left(\prod_{i \in S^{*}} \mathrm{P}\left\{X_{i}=0\right\}\right) \sum_{s_{m} \leq i_{1}<\cdots<i_{j} \leq n}\left(\prod_{i \in\left\{i_{1}, \ldots, i_{j}\right\}} \frac{\mathrm{P}\left\{X_{i}=1\right\}}{\mathrm{P}\left\{X_{i}=0\right\}}\right) \\
& =\left(\prod_{i=s_{m}}^{n} q_{i}\right) R_{s_{m}, j},
\end{aligned}
$$

which, combined with (2.11), proves (1.3) of the STMOT.

\section{Application to the secretary problem}

The secretary problem can be described as follows. A known number, $n$, of rankable applicants ( 1 being the best and $n$ the worst) appear one at a time in a random order, with all $n$ ! permutations equally likely. That is, each of the successive ranks of $n$ applicants constitutes a random permutation. Suppose that all that can be observed are the relative ranks of the applicants as they appear. If $Y_{j}$ denotes the relative rank of the $j$ th applicant among the first $j$ applicants, the sequentially observed random variables are $Y_{1}, Y_{2}, \ldots, Y_{n}$. It is well known that

(a) $Y_{1}, Y_{2}, \ldots, Y_{n}$ are independent random variables;

(b) $\mathrm{P}\left\{Y_{j}=i\right\}=1 / j, 1 \leq i \leq j, 1 \leq j \leq n$.

The $j$ th applicant is called a candidate if he/she is relatively best, i.e. $Y_{j}=1$. The problem we consider here is to stop on any of the last $m$ successes, that is, any of the last $m$ candidates (stopping is identified with selection of an applicant in the secretary problem). The independent random variables of Section 1 are therefore $X_{1}, X_{2}, \ldots, X_{n}$, where $X_{j}=\mathbf{1}\left(Y_{j}=1\right)$ from (a). Since $p_{j}=\mathrm{P}\left\{X_{j}=1\right\}=1 / j$ and so $r_{j}=1 /(j-1)$ from (b), we immediately have the following result from the STMOT.

Lemma 3.1. For the secretary problem, the optimal rule passes up the first $s_{m}-1$ applicants and then selects the first candidate, if any, where

$$
s_{m}=\min \left\{k \geq 1: \sum_{k+1 \leq i_{1}<i_{2}<\cdots<i_{m} \leq n} \prod_{j=1}^{m} \frac{1}{i_{j}-1} \leq 1\right\} .
$$

The maximal win probability is

$$
v_{m}=\frac{s_{m}-1}{n} \sum_{k=1}^{m}\left(\sum_{s_{m} \leq i_{1}<i_{2}<\cdots<i_{k} \leq n} \prod_{j=1}^{k} \frac{1}{i_{j}-1}\right) .
$$


For small values of $n, s_{m}$ and $v_{m}$ can easily be computed from (3.1) and (3.2), respectively. For example, we have, for $m=2$,

$$
\begin{array}{lll}
s_{2}=2 & \text { and } & v_{2}=\frac{17}{24} \approx 0.708 \text { for } n=5, \\
s_{2}=3 & \text { and } & v_{2}=\frac{1303}{2016} \approx 0.646 \text { for } n=10 .
\end{array}
$$

Of interest are the approximate values of $s_{m}$ and $v_{m}$ for large $n$.

Lemma 3.2. Let $n$ tend to $\infty$. Then, asymptotically,

$$
\begin{aligned}
& \text { (i) } s_{m}^{*}=\lim _{n \rightarrow \infty} \frac{s_{m}}{n}=\exp \left\{-(m !)^{1 / m}\right\}, \\
& \text { (ii) } v_{m}^{*}=\lim _{n \rightarrow \infty} v_{m}=\exp \left\{-(m !)^{1 / m}\right\} \sum_{j=1}^{m} \frac{(m !)^{j / m}}{j !} .
\end{aligned}
$$

Proof. We give an intuitive proof. If we let $n$ tend to $\infty$ and write $s$ as the limit of $k / n$, then, using $t_{j}$ for $i_{j} / n$ and $\mathrm{d} t_{j}$ for $1 / n, 1 \leq j \leq m$, the sum in (3.1) becomes a Riemann approximation to a multiple integral,

$$
\begin{aligned}
& \sum_{k+1 \leq i_{1}<i_{2}<\cdots<i_{m} \leq n}\left(\frac{1}{i_{1}-1}\right)\left(\frac{1}{i_{2}-1}\right) \cdots\left(\frac{1}{i_{m}-1}\right) \\
& \rightarrow \int_{s \leq t_{1}<t_{2}<\cdots<t_{m} \leq 1}\left(\frac{\mathrm{d} t_{1}}{t_{1}}\right)\left(\frac{\mathrm{d} t_{2}}{t_{2}}\right) \cdots\left(\frac{\mathrm{d} t_{m}}{t_{m}}\right) \\
= & \int_{s}^{1} \frac{\mathrm{d} t_{1}}{t_{1}} \int_{t_{1}}^{1} \frac{\mathrm{d} t_{2}}{t_{2}} \cdots \int_{t_{m-1}}^{1} \frac{\mathrm{d} t_{m}}{t_{m}} \\
= & \frac{(-\log s)^{m}}{m !} .
\end{aligned}
$$

Equating this to 1 yields $s=s_{m}^{*}$ and proves (i) from (3.1). In a similar way, we have, from (3.2),

$$
v_{m} \rightarrow v_{m}^{*}=\left(s_{m}^{*}\right) \sum_{j=1}^{m} \int_{s_{m}^{*}}^{1} \frac{\mathrm{d} t_{1}}{t_{1}} \int_{t_{1}}^{1} \frac{\mathrm{d} t_{2}}{t_{2}} \cdots \int_{t_{j-1}}^{1} \frac{\mathrm{d} t_{j}}{t_{j}}=\left(s_{m}^{*}\right) \sum_{j=1}^{m} \frac{\left(-\log s_{m}^{*}\right)^{j}}{j !},
$$

which gives (ii) via (i).

Table 1 presents some numerical values of $s_{m}^{*}$ and $v_{m}^{*}$ for given $m$. The case in which $m=1$ is the classical secretary problem studied in Lindley (1961), Dynkin (1963), and Gilbert and Mosteller (1966). Our problem seems similar to that of choosing one of the $m$ best considered in Gusein-Zade (1966), Gilbert and Mosteller (1966), and Frank and Samuels (1980). However, these two problems are different because the $k$ th last candidate is not necessarily the $k$ th best

TABLE 1: Values of $s_{m}^{*}$ and $v_{m}^{*}$ for several $m$.

\begin{tabular}{ccccccc}
\hline$m$ & 1 & 2 & 3 & 4 & 5 & 10 \\
\hline$s_{m}^{*}$ & 0.3679 & 0.2431 & 0.1625 & 0.1093 & 0.0739 & 0.0108 \\
$v_{m}^{*}$ & 0.3679 & 0.5869 & 0.7260 & 0.8167 & 0.8767 & 0.9822 \\
\hline
\end{tabular}


among all candidates for $k \geq 2$, and the only information available at each stage in our problem is record (candidate) information and not (relative) rank information. The reader is referred to Ferguson (1989) and Samuels (1991) for reviews of the secretary problem.

We can easily obtain further asymptotic quantities of interest by using a nonhomogeneous Poisson process (NPP) model. Presman and Sonin (1972, p. 772) found the NPP to be an appropriate setting in which to define the infinite version of the secretary problem as the limit of the corresponding finite problems. This model facilitates the derivation of the asymptotic values. Suppose that $n$ applicants appear at fractional times $1 / n, 2 / n, \ldots, n / n$ instead of at times $1,2, \ldots, n$. As $k / n \rightarrow x$ with $n \rightarrow \infty$, we can find from properties (a) and (b) that the occurrence of candidates in the limiting problem constitutes an NPP with intensity function $\lambda(x)=1 / x, 0<x \leq 1$. That is, if we denote by $M(s, t)$ the number of candidates appearing in the time interval $(s, t), 0<s<t \leq 1$, then

$$
\mathrm{P}\{M(s, t)=k\}=\mathrm{e}^{-\Lambda(s, t)} \frac{\{\Lambda(s, t)\}^{k}}{k !}, \quad k \geq 0,
$$

where

$$
\Lambda(s, t)=\int_{s}^{t} \lambda(x) \mathrm{d} x=\log \left(\frac{t}{s}\right) .
$$

This can be seen as a refinement of Theorem 1 of Gilbert and Mosteller (1966). Let $f(x)$ be the win probability when we use a (limiting) threshold rule with value $x$, which stops on the first candidate that appears after time $x$, if any. Then, from (3.3),

$$
f(x)=\sum_{k=1}^{m} \mathrm{P}\{M(x, 1)=k\}=\sum_{k=1}^{m} \frac{x(-\log x)^{k}}{k !} .
$$

It is easy to see that $f(x)$ is maximized at $x=s_{m}^{*}$ and yields $f\left(s_{m}^{*}\right)=v_{m}^{*}$, as desired from Lemma 3.2. Note that the value $s_{m}^{*}$ represents not only the optimal threshold value but also the probability of no choice (no stop), i.e. the probability that no candidate appears until the very end, which follows from $\mathrm{P}\left\{M\left(s_{m}^{*}, 1\right)=0\right\}=s_{m}^{*}$. Since $M\left(s_{m}^{*}, 1\right)$ is a Poisson random variable with mean $\Lambda\left(s_{m}^{*}, 1\right)=-\log s_{m}^{*}=(m !)^{1 / m}$, we have the following result.

Lemma 3.3. (i) The expected number of candidates that appear after $s_{m}^{*}$ is a geometric mean of $m$ integers $1,2, \ldots, m$, that is,

$$
\mathrm{E}\left[M\left(s_{m}^{*}, 1\right)\right]=(m !)^{1 / m} .
$$

(ii) The probability of stopping on the kth last candidate conditional on the win being achieved is

$$
\frac{(m !)^{k / m}}{k !} / \sum_{j=1}^{m} \frac{(m !)^{j / m}}{j !}, \quad 1 \leq k \leq m .
$$

(iii) Let $K_{m}$ be the expected rank of the applicant chosen conditional on stopping. Then

$$
K_{m}=\frac{1}{2}\left[1+\exp \left\{(m !)^{1 / m}\right\}\right]
$$


Proof. Part (i) is immediate from $\mathrm{E}\left[M\left(s_{m}^{*}, 1\right)\right]=\Lambda\left(s_{m}^{*}, 1\right)$. Part (ii) follows because this conditional probability is just

$$
\frac{\mathrm{P}\left\{M\left(s_{m}^{*}, 1\right)=k\right\}}{\sum_{j=1}^{m} \mathrm{P}\left\{M\left(s_{m}^{*}, 1\right)=j\right\}} .
$$

To prove part (iii), let $T_{m}$ be the stopping time of the optimal rule, i.e. the arrival time of the first candidate after $s_{m}^{*}$, if any. If no candidate appears, $T_{m}$ is defined to be 1 . Since $T_{m}>t$ is equivalent to $M\left(s_{m}^{*}, t\right)=0$ for $t<1$, the density of $T_{m}$ is

$$
f_{T_{m}}(t)=-\frac{\mathrm{d}}{\mathrm{d} t} \mathrm{P}\left\{T_{m}>t\right\}=-\frac{\mathrm{d}}{\mathrm{d} t}\left(\frac{s_{m}^{*}}{t}\right)=\frac{s_{m}^{*}}{t^{2}} .
$$

Obviously, $\mathrm{P}\left\{T_{m}=1\right\}=s_{m}^{*}$. We only stop on a candidate, i.e. relatively best applicant, so the expected rank of the applicant chosen at time $t$ is $1 / t$ (see, e.g. Mucci (1973)). Hence,

$$
K_{m}=\int_{s_{m}^{*}}^{1} \frac{1}{t} \frac{f_{T_{m}}(t)}{1-\mathrm{P}\left\{T_{m}=1\right\}} \mathrm{d} t
$$

which yields (iii).

For example, $K_{1}=1.859, K_{2}=2.557, K_{3}=3.577, K_{4}=5.073$, and $K_{5}=7.267$. These show that, for small $m$, the optimal rule chooses a pretty good applicant though it has probability $s_{m}^{*}$ of no choice as a risk. As for the rank minimization problem whose objective is to minimize the expected rank of the applicant chosen with the severe condition that one applicant must be chosen, Chow et al. (1964) showed that the minimal asymptotic expected rank is approximately 3.869. See also Krieger and Samuel-Cahn (2009). We can give the arrival time distribution of the $k$ th last candidate.

Lemma 3.4. Let $Z_{k}$ denote the arrival time of the $k$ th last candidate. Then the density function of $Z_{k}, k \geq 1$, is

$$
f_{k}(z)=\frac{(-\log z)^{k-1}}{(k-1) !}, \quad 0<z<1 .
$$

Proof. Observe that the event $Z_{k} \leq z$ occurs if and only if $M(z, 1) \leq k-1$ for each $k$. Hence,

$$
f_{k}(z)=\frac{\mathrm{d}}{\mathrm{d} z} \mathrm{P}\left\{Z_{k} \leq z\right\}=\frac{\mathrm{d}}{\mathrm{d} z}\left(\sum_{j=0}^{k-1} \mathrm{P}\{M(z, 1)=j\}\right)=\frac{(-\log z)^{k-1}}{(k-1) !}
$$

which completes the proof.

For later use, we return to the finite problem and review the distribution of the number of candidates. Define

$$
M_{n}=X_{1}+X_{2}+\cdots+X_{n}
$$

where $X_{j}=\mathbf{1}\left(Y_{j}=1\right)$ is as defined at the beginning of this section. Then $M_{n}$ denotes the total number of candidates. It is well known (see, e.g. Equation (2.5.9) of Arnold et al. (1998) or Sections 6.2, 6.3, and 9.5 of Blom et al. (1994)) that the probability mass function of $M_{n}$ is expressed as

$$
p_{n}(k)=\mathrm{P}\left\{M_{n}=k\right\}=\frac{1}{n !}\left[\begin{array}{l}
n \\
k
\end{array}\right], \quad 1 \leq k \leq n,
$$


where the notation $\left[\begin{array}{l}n \\ k\end{array}\right], 1 \leq k \leq n, 1 \leq n$, is a real number called the Stirling number of the first kind (see Knuth (1992) for this notation). This number can be simply calculated from the following recursive relation:

$$
\left[\begin{array}{l}
n \\
k
\end{array}\right]=\left[\begin{array}{l}
n-1 \\
k-1
\end{array}\right]+(n-1)\left[\begin{array}{c}
n-1 \\
k
\end{array}\right], \quad 1 \leq k \leq n, 2 \leq n,
$$

with $\left[\begin{array}{l}1 \\ 1\end{array}\right]=1$ and $\left[\begin{array}{l}n \\ k\end{array}\right]=0$ for $k=0$ or $k>n$. Therefore, $p_{n}(k)$ satisfies the recursion

$$
p_{n}(k)=\frac{1}{n} p_{n-1}(k-1)+\left(1-\frac{1}{n}\right) p_{n-1}(k), \quad 1 \leq k \leq n, 2 \leq n,
$$

with $p_{1}(1)=1$ and $p_{n}(k)=0$ for $k=0$ or $k>n$.

\section{Full-information analogue}

In contrast to the no-information problem considered in Section 3, the full-information analogue is the problem in which the observations are the true values of $n$ applicants $Y_{1}, Y_{2}, \ldots, Y_{n}$, assumed to be independent and identically distributed random variables from a known continuous distribution, taken without loss of generality to be the uniform distribution on the interval $[0,1]$. Let $L_{k}=\max \left\{Y_{1}, Y_{2}, \ldots, Y_{k}\right\}$ be the maximum of the first $k$ observations and call the $k$ th observation or the $k$ th applicant a record if $L_{k}=Y_{k}$. It is desired to obtain a stopping rule that maximizes the probability of stopping on any of the last $m$ successes, that is, the last $m$ records. The case in which $m=1$ is the full-information best-choice problem solved in Gilbert and Mosteller (1966). For ease of description, let $a_{k}=\mathrm{P}\left\{M_{k}<m\right\}, k \geq 0$, be the probability that the number of candidates is less than $m$ when the total number of applicants is $k$ in the secretary problem, namely, $a_{k}=\sum_{i=1}^{m-1} p_{k}(i)$ for $k \geq m$ and $a_{k}=1$ for $k<m\left(a_{0}=1\right.$ for convenience). The main results can be summarized as follows.

Theorem 4.1. (a) Optimal stopping rule: for a given positive integer $m$, there exists a nondecreasing sequence of the thresholds $\left\{b_{j}(m), 1 \leq j\right\}$ defined as $b_{j}(m)=0$ for $1 \leq j<m$ and as a unique solution $x \in(0,1)$ to the equation

$$
\sum_{i=m}^{j} p_{i}(m)\left(\begin{array}{l}
j \\
i
\end{array}\right)\left(\frac{1-x}{x}\right)^{i}=1
$$

for $j \geq m$, such that the optimal rule is to choose the first record $Y_{k}\left(=L_{k}\right)$ that exceeds the threshold $b_{n-k}(m)$. Henceforth, we simply write $b_{j}$ for $b_{j}(m)$ unless otherwise specified.

(b) Optimal probability: let $P_{n, m}^{*}$ denote the optimal win probability as a function of $n$ and $m$; then

$$
P_{n, m}^{*}=\sum_{r=1}^{n} P(r)
$$

where

$$
P(1)=\frac{1}{n} \sum_{k=0}^{n-1} a_{k}\left(\begin{array}{c}
n-1 \\
k
\end{array}\right) \sum_{j=k+1}^{n}\left(\begin{array}{l}
n \\
j
\end{array}\right)\left(1-b_{n-1}\right)^{j} b_{n-1}^{n-j}
$$

and, for $2 \leq r \leq n$,

$$
P(r)=\frac{1}{r-1} \sum_{k=0}^{n-r} a_{k}\left(\begin{array}{c}
n-r \\
k
\end{array}\right) \sum_{i=1}^{r-1}\left[P_{1}(i, k)+P_{2}(i, k)\right],
$$


with

$$
P_{1}(i, k)=\sum_{j=k+1}^{n} \frac{\left(\begin{array}{l}
n \\
j
\end{array}\right)}{\left(\begin{array}{l}
n \\
k
\end{array}\right)} \frac{\left(1-b_{n-r}\right)^{j} b_{n-r}^{n-j}-\left(1-b_{n-i}\right)^{j} b_{n-i}^{n-j}}{n-k}
$$

and

$$
P_{2}(i, k)=\sum_{j=k+1}^{n-r+1} \frac{\left(\begin{array}{c}
n-r+1 \\
j
\end{array}\right)}{\left(\begin{array}{c}
n-r+1 \\
k
\end{array}\right)} \frac{\left(1-b_{n-i}\right)^{j} b_{n-i}^{n-j}}{n-r-k+1} .
$$

(c) Asymptotics: let $c_{m}$ be the unique root $t$ to the equation

$$
\sum_{i=m}^{\infty} p_{i}(m) \frac{t^{i}}{i !}=1
$$

then, as $n \rightarrow \infty$,

$$
P_{n, m}^{*} \rightarrow P_{m}^{*}=\mathrm{e}^{-c_{m}} J_{m}\left(c_{m}\right)+\left\{K_{m}\left(c_{m}\right)-c_{m} J_{m}\left(c_{m}\right)\right\} I\left(c_{m}\right),
$$

where

$$
\begin{aligned}
I(t) & =\int_{1}^{\infty} \frac{\mathrm{e}^{-t x}}{x} \mathrm{~d} x, \\
J_{m}(t) & =\sum_{j=0}^{\infty} a_{j} \frac{t^{j}}{j !}=\sum_{j=0}^{m-1} \frac{t^{j}}{j !}+\sum_{j=m}^{\infty} a_{j} \frac{t^{j}}{j !}, \\
K_{m}(t) & =\sum_{i=1}^{\infty} \min (i, m) \frac{t^{i}}{i !}+\sum_{i=m+1}^{\infty}\left(\sum_{j=m}^{i-1} a_{j}\right) \frac{t^{i}}{i !} .
\end{aligned}
$$

Proof. (a) In Corollary 2.1, the Bernoulli random variables are $X_{1}, X_{2}, \ldots, X_{n}$, where $X_{k}=\mathbf{1}\left(Y_{k}=L_{k}\right)$, and the $\sigma$-field $\mathcal{F}_{k}$ is the $\sigma$-field generated by the variables $Y_{1}, Y_{2}, \ldots, Y_{k}$. We easily see that

$$
V_{k}=\left(L_{k}\right)^{n-k} \text {. }
$$

On the other hand, we may compute $W_{k}$ by conditioning on the number, $J$, of future observations whose values are greater than $L_{k}$. Here $J$ is a binomial random variable with parameters $\left(n-k, 1-L_{k}\right)$, i.e.

$$
\mathrm{P}\{J=j\}=\left(\begin{array}{c}
n-k \\
j
\end{array}\right)\left(1-L_{k}\right)^{j} L_{k}^{n-k-j},
$$

and, moreover, given $J=j$, the probability that the number of future records (candidates) is $m$ is $p_{j}(m)$ by exchangeability. Thus,

$$
W_{k}=\sum_{j=m}^{n-k} p_{j}(m)\left(\begin{array}{c}
n-k \\
j
\end{array}\right)\left(1-L_{k}\right)^{j} L_{k}^{n-k-j},
$$

and, hence,

$$
\frac{W_{k}}{V_{k}}=\sum_{j=m}^{n-k} p_{j}(m)\left(\begin{array}{c}
n-k \\
j
\end{array}\right)\left(\frac{1-L_{k}}{L_{k}}\right)^{j}
$$


for $k \leq n-m$ and 0 otherwise. Since the nondecreasing nature of $L_{k}$ implies that

$$
\frac{1-L_{k}}{L_{k}} \geq \frac{1-L_{k+1}}{L_{k+1}} \text { a.s., }
$$

we have, for $k \leq n-m-1$,

$$
\frac{W_{k}}{V_{k}}-\frac{W_{k+1}}{V_{k+1}} \geq \sum_{j=m}^{n-k-1} p_{j}(m)\left(\begin{array}{c}
n-k-1 \\
j-1
\end{array}\right)\left(\frac{1-L_{k}}{L_{k}}\right)^{j} \geq 0,
$$

which shows that condition (2.8) is satisfied. Hence, the 1-sla rule, $N_{m}$ of (2.7), is optimal and written as, from (4.6) and the definition of the threshold, (4.1),

$$
N_{m}=\min \left\{k \geq 1: Y_{k}=L_{k} \geq b_{n-k}\right\} .
$$

(b) Let $P(r)$ be the win probability when stopping on the $r$ th observation. If we denote by $W$ the win event then, conditioning on the $r$ th observation being a record with value $x$, i.e. $L_{r}=Y_{r}=x$, we can express

$$
P(r)=\int_{b_{n-r}}^{1} \mathrm{P}\left\{W \mid L_{r}=Y_{r}=x\right\} \frac{1}{r} f_{r}(x) \mathrm{d} x,
$$

where $f_{r}(x)=r x^{r-1}$ is a density of $L_{r}$. We first consider the case in which $r \geq 2$. Note that, since the threshold $b_{j}$ is nondecreasing, stopping on the $r$ th observation leads to $W$ if and only if

(i) the previous maximum is less than the corresponding threshold, i.e. $L_{r-1}<b_{n-i}$ if $L_{r-1}=Y_{i}, 1 \leq i \leq r-1$;

(ii) the number of future records is less than $m$.

Let $A$ and $B$ denote the events in (i) and (ii), respectively. Then it is easy to see that $A$ and $B$ are conditionally independent given $L_{r}=Y_{r}=x$, so

$$
\mathrm{P}\left\{W \mid L_{r}=Y_{r}=x\right\}=\mathrm{P}\left\{A \mid L_{r}=Y_{r}=x\right\} \mathrm{P}\left\{B \mid L_{r}=Y_{r}=x\right\} .
$$

Given $L_{r}=Y_{r}=x, L_{r-1}$ can be viewed as the maximum of $r-1$ independent random variables each uniformly distributed on $(0, x)$, and the arrival time of the previous maximum is uniform on $\{1,2, \ldots, r-1\}$ independently of $L_{r-1}$. Hence,

$$
\mathrm{P}\left\{A \mid L_{r}=Y_{r}=x\right\}=\frac{1}{r-1} \sum_{i=1}^{r-1}\left[\frac{\min \left(x, b_{n-i}\right)}{x}\right]^{r-1} .
$$

On the other hand, we have

$$
\mathrm{P}\left\{B \mid L_{r}=Y_{r}=x\right\}=\sum_{k=0}^{n-r} a_{k}\left(\begin{array}{c}
n-r \\
k
\end{array}\right)(1-x)^{k} x^{n-r-k},
$$


using a similar reasoning to that used to derive $W_{k}$ in (a). Therefore, we have, from (4.7)-(4.9),

$$
\begin{aligned}
P(r)= & \frac{1}{r-1} \sum_{k=0}^{n-r} a_{k}\left(\begin{array}{c}
n-r \\
k
\end{array}\right) \\
& \times \sum_{i=1}^{r-1}\left\{\int_{b_{n-r}}^{b_{n-i}} x^{n-k-1}(1-x)^{k} \mathrm{~d} x+b_{n-i}^{r-1} \int_{b_{n-i}}^{1} x^{n-k-r}(1-x)^{k} \mathrm{~d} x\right\},
\end{aligned}
$$

which gives (4.3) via the well-known formula

$$
\int_{c}^{1} x^{m-1}(1-x)^{n-1} \mathrm{~d} x=\sum_{j=n}^{m+n-1} \frac{\left(\begin{array}{c}
m+n-1 \\
j
\end{array}\right)}{\left(\begin{array}{c}
m+n-1 \\
n-1
\end{array}\right)} \frac{(1-c)^{j} c^{m+n-1-j}}{m}
$$

for $0<c \leq 1$ and positive integers $m$ and $n$. The case in which $r=1$ is special. Stopping on the first observation leads to $W$ if and only if the number of future records is less than $m$. Hence, substituting

$$
\mathrm{P}\left\{W \mid L_{1}=Y_{1}=x\right\}=\sum_{k=0}^{n-1} a_{k}\left(\begin{array}{c}
n-1 \\
k
\end{array}\right)(1-x)^{k} x^{n-1-k}
$$

into (4.7) yields (4.2).

(c) To derive the asymptotic result (4.5), we can use a planar Poisson process (PPP) model which is known to facilitate the derivation of the asymptotic values for some full-information problems (see, e.g. Gnedin (1996), (2004), Samuels (2004), Bruss and Swan (2009), and Tamaki (2009)). According to Samuels (2004, Sections 9 and 10), we use a Poisson process with unit rate on the semi-infinite strip $[0,1] \times[0, \infty)$. This turns the problem upside down, making the 'best' become the 'smallest'. The process is scanned from left to right by shifting a vertical detector and the scanning can be stopped each time a point in the PPP, referred to as an atom henceforth, is detected. A link to the finite problems can be established by suitably embedding the finite independent and identically distributed sequences in the PPP in a similar manner as given for the Gilbert and Mosteller full-information best-choice problem in Gnedin (1996, Section 3).

Suppose that an atom is identified as a point $(t, y)$ if the atom appears at time $t$ as a record (the relatively best atom as in the finite problem) having value $y$ in the PPP. Let $Q_{k}(t, y)$ denote the probability that $k$ records appear in the future after skipping the point $(t, y)$. Then if we denote by $\mathrm{P}_{\mathrm{Poisson}}(j, \mu)$ the Poisson probability of $j$ events for a given mean $\mu$, i.e. $\mathrm{P}_{\text {Poisson }}(j, \mu)=\mathrm{e}^{-\mu}(\mu)^{j} / j$ !, we have

$$
Q_{k}(t, y)=\sum_{j=k}^{\infty} p_{j}(k) \mathrm{P}_{\text {Poisson }}(j, y(1-t)), \quad k \geq 0,
$$

because $\mathrm{P}_{\mathrm{Poisson}}(j, y(1-t))$ is the probability that there exist $j$ atoms in the box domain $[t, 1] \times[0, y]$ whose area is $y(1-t)$ and, conditional on there being $j$ atoms, the probability that $k$ of them are records is $p_{j}(k)$ by exchangeability.

Let, in particular,

$$
V(t, y)=Q_{0}(t, y), \quad W(t, y)=Q_{m}(t, y) .
$$


Solving for the locus of point $(t, y)$ at which the win probability, if we stop immediately, is equal to the win probability when stopping on the next record, if any, or, equivalently,

$$
\sum_{k=0}^{m-1} Q_{k}(t, y)=\sum_{k=1}^{m} Q_{k}(t, y),
$$

yields $W(t, y) / V(t, y)=1$, which implies that $y(1-t)=c_{m}$, where $c_{m}$, is defined by (4.4) (for simplicity, we write $c$ for $c_{m}$ throughout the proof). Since $W(t, y) / V(t, y) \leq 1$ implies that $W\left(t^{\prime}, y^{\prime}\right) / V\left(t^{\prime}, y^{\prime}\right) \leq 1$ for $t^{\prime}>t, y^{\prime}<y$, we are in the monotone case of optimal stopping (continuous analogue of Corollary 2.1) and we can conclude that the optimal rule stops with the first record, if any, that lies below the threshold curve $y=c /(1-t)$. Let $T$ be the arrival time of the first (leftmost) atom that lies below the threshold curve $y=c /(1-t)$, and let $S$ be the time when the value of the best (lowest) atom above the threshold is equal to the threshold. Then $T$ and $S$ are independent and their distributions are given by

$$
\begin{aligned}
& \mathrm{P}\{T>t\}=\mathrm{P}_{\text {Poisson }}\left(0, \int_{0}^{t} g(r) \mathrm{d} r\right), \\
& \mathrm{P}\{S>s\}=\mathrm{P}_{\text {Poisson }}\left(0, \int_{0}^{s}(g(s)-g(r)) \mathrm{d} r\right),
\end{aligned}
$$

where $g(r)=c /(1-r), 0<r<1$.

Considering that the win probability when we stop at point $(t, y)$ is

$$
u(t, y)=\sum_{k=0}^{m-1} Q_{k}(t, y),
$$

we have, by exploiting the virtual stopping time $\min (S, T)$ which makes the calculations simpler,

$$
\begin{aligned}
P_{m}^{*}= & \int_{0}^{1} \int_{0}^{t} u\left(s, \frac{c}{1-s}\right) f_{S}(s) f_{T}(t) \mathrm{d} s \mathrm{~d} t \\
& +\int_{0}^{1} \int_{0}^{s}\left(\frac{1-t}{c} \int_{0}^{c /(1-t)} u(t, y) \mathrm{d} y\right) f_{T}(t) f_{S}(s) \mathrm{d} t \mathrm{~d} s
\end{aligned}
$$

where $f_{T}(t)$ and $f_{S}(s)$ are the densities of $T$ and $S$, respectively.

We have, from (4.13) and (4.10),

$$
\begin{aligned}
u\left(s, \frac{c}{1-s}\right)= & \sum_{k=0}^{m-1} \sum_{j=k}^{\infty} p_{j}(k) \mathrm{P}_{\text {Poisson }}(j, c) \\
= & \sum_{j=0}^{m-1}\left(\sum_{k=0}^{j} p_{j}(k)\right) \mathrm{P}_{\text {Poisson }}(j, c) \\
& +\sum_{j=m}^{\infty}\left(\sum_{k=0}^{m-1} p_{j}(k)\right) \mathrm{P}_{\text {Poisson }}(j, c) \\
= & \sum_{j=0}^{m-1} \mathrm{P}_{\text {Poisson }}(j, c)+\sum_{j=m}^{\infty} a_{j} \mathrm{P}_{\text {Poisson }}(j, c) \\
= & \mathrm{e}^{-c} J_{m}(c) .
\end{aligned}
$$


Similarly,

$$
\begin{aligned}
\int_{0}^{c /(1-t)} u(t, y) \mathrm{d} y & =\sum_{k=0}^{m-1} \sum_{j=k}^{\infty} p_{j}(k) \int_{0}^{c /(1-t)} \mathrm{e}^{-y(1-t)} \frac{[y(1-t)]^{j}}{j !} \mathrm{d} y \\
& =(1-t)^{-1} \sum_{k=0}^{m-1} \sum_{j=k}^{\infty} p_{j}(k) \int_{0}^{c} \mathrm{e}^{-x} \frac{x^{j}}{j !} \mathrm{d} x \\
& =(1-t)^{-1} \sum_{k=0}^{m-1} \sum_{j=k}^{\infty} p_{j}(k)\left(\sum_{i=j+1}^{\infty} \mathrm{P}_{\text {Poisson }}(i, c)\right),
\end{aligned}
$$

where the last equality follows from the well-known identity which states that, for any $c>0$, the event 'there are more than $j$ arrivals by time $c$ ' is the same as the event 'the waiting time for the $(j+1)$ th arrival is less than $c^{\prime}$. However, interchanging the order of summation successively, we have

$$
\begin{aligned}
\sum_{k=0}^{m-1} \sum_{j=k}^{\infty} p_{j}(k)\left(\sum_{i=j+1}^{\infty} \mathrm{P}_{\text {Poisson }}(i, c)\right) & \\
= & \sum_{k=0}^{m-1} \sum_{i=k+1}^{\infty}\left(\sum_{j=k}^{i-1} p_{j}(k)\right) \mathrm{P}_{\text {Poisson }}(i, c) \\
= & \sum_{i=1}^{m}\left[\sum_{k=0}^{i-1}\left(\sum_{j=k}^{i-1} p_{j}(k)\right)\right] \mathrm{P}_{\text {Poisson }}(i, c)+\sum_{i=m+1}^{\infty}\left[\sum_{k=0}^{m-1}\left(\sum_{j=k}^{i-1} p_{j}(k)\right)\right] \mathrm{P}_{\text {Poisson }}(i, c) \\
= & \sum_{i=1}^{m}\left[\sum_{j=0}^{i-1}\left(\sum_{k=0}^{j} p_{j}(k)\right)\right] \mathrm{P}_{\text {Poisson }}(i, c) \\
& +\sum_{i=m+1}^{\infty}\left[\sum_{j=0}^{m-1}\left(\sum_{k=0}^{j} p_{j}(k)\right)+\sum_{j=m}^{i-1}\left(\sum_{k=0}^{m-1} p_{j}(k)\right)\right] \mathrm{P}_{\text {Poisson }}(i, c) \\
= & \sum_{i=1}^{m}{ }_{i} \mathrm{P}_{\text {Poisson }}(i, c)+\sum_{i=m+1}^{\infty}\left(m+\sum_{j=m}^{i-1} a_{j}\right) \mathrm{P}_{\text {Poisson }}(i, c) \\
= & \mathrm{e}^{-c} K_{m}(c) .
\end{aligned}
$$

Substituting the three expressions (4.15)-(4.17) into (4.14), we obtain

$$
\begin{aligned}
P_{m}^{*} & =\mathrm{e}^{-c} J_{m}(c) \mathrm{P}\{S \leq T\}+\mathrm{e}^{-c} c^{-1} K_{m}(c) \mathrm{P}\{S>T\} \\
& =\mathrm{e}^{-c} J_{m}(c)+\left(K_{m}(c)-c J_{m}(c)\right) \mathrm{e}^{-c} c^{-1} \mathrm{P}\{S>T\} .
\end{aligned}
$$

Note that we have, from (4.11) and (4.12),

$$
\begin{aligned}
\mathrm{P}\{S>T\} & =\int_{0}^{1} \mathrm{P}\{S>t\} f_{T}(t) \mathrm{d} t \\
& =\int_{0}^{1}(1-t)^{-c} \mathrm{e}^{-c t /(1-t)} c(1-t)^{c-1} \mathrm{~d} t \\
& =c \mathrm{e}^{c} I(c),
\end{aligned}
$$

which, combined with (4.18), gives the desired result (4.5). Thus, the proof is complete. 
TABLE 2: Values of $c_{m}$ and $P_{m}^{*}$ for several $m$.

\begin{tabular}{ccccccr}
\hline$m$ & 1 & 2 & 3 & 4 & 5 & 10 \\
\hline$c_{m}$ & 0.8044 & 1.5151 & 2.3731 & 3.3573 & 4.4523 & 11.2433 \\
$P_{m}^{*}$ & 0.5802 & 0.8424 & 0.9465 & 0.9834 & 0.9953 & 0.9999 \\
\hline
\end{tabular}

Table 2 presents some numerical values of $c_{m}$ and $P_{m}^{*}$.

Remark 4.1. Since $p_{n}(n)=1 / n$ !, (4.1) for $j=m$ can be written as

$$
\frac{1}{m !}\left(\frac{1-x}{x}\right)^{m}=1
$$

which yields

$$
b_{m}(m)=\frac{1}{1+(m !)^{1 / m}} .
$$

Similarly, considering that

$$
p_{n}(n-1)=\frac{1}{2 \cdot(n-2) !}, \quad p_{n}(n-2)=\frac{3 n-1}{24 \cdot(n-3) !},
$$

which can be obtained from the recursive formula (3.4), we have, as another expression of (4.1) for $j=m+1$ and $j=m+2$,

$$
\frac{m+1}{m !}\left(\frac{1-x}{x}\right)^{m}+\frac{1}{2 \cdot(m-1) !}\left(\frac{1-x}{x}\right)^{m+1}=1
$$

and

$$
\frac{(m+1)(m+2)}{2 \cdot m !}\left(\frac{1-x}{x}\right)^{m}+\frac{m+2}{2 \cdot(m-1) !}\left(\frac{1-x}{x}\right)^{m+1}+\frac{3 m+5}{24 \cdot(m-1) !}\left(\frac{1-x}{x}\right)^{m+2}=1,
$$

respectively. In particular, from $(4.19), b_{2}(1)=(1+\sqrt{6}) / 5$ and $b_{3}(2)=\sqrt{3} / 3$.

\section{Acknowledgement}

The author is grateful to an anonymous referee for his/her careful reading.

\section{References}

Arnold, B. C., Barlakrishnan, N. and Nagaraja, H. N. (1998). Records. John Wiley, New York.

Blom, G., Holst, L. AND Sandel, D. (1994). Problems and Snapshots from the World of Probability. Springer, New York.

Bruss, F. T. (2000). Sum the odds to one and stop. Ann. Prob. 28, 1384-1391.

Bruss, F. T. (2003). A note on bounds for the odds theorem of optimal stopping. Ann. Prob. 31, 1859-1861.

Bruss, F. T. AND Louchard, G. (2009). The odds algorithm based on sequential updating and its performance. Adv. Appl. Prob. 41, 131-153.

Bruss, F. T. And Paindaveine, D. (2000). Selecting a sequence of last successes in independent trials. J. Appl. Prob. 37, 389-399.

Bruss, F. T. AND SWAN, Y. C. (2009). A continuous-time approach to Robbins' problem of minimizing the expected rank. J. Appl. Prob. 46, 1-18.

Chow, Y. S., Robbins, H. And Siegmund, D. (1971). Great Expectations: The Theory of Optimal Stopping. Houghton Mifflin, Boston, MA. 
Chow, Y. S., Moriguti, S., Robbins, H. and Samuels, S. M. (1964). Optimal selection based on relative rank (the 'secretary problem'). Israel J. Math. 2, 81-90.

Dynkin, E. B. (1963). The optimal choice of the instant for stopping a Markov process. Soviet Math. Dokl. 4, $627-629$.

FERGUSON, T. S. (1989). Who solved the secretary problem? Statist. Sci. 4, 282-289.

Ferguson, T. S. (2006). Optimal Stopping and Applications. Available at http://www.math.ucla.edu/ tom/Stopping/ Contents.html.

FERguson, T. S. (2008). The sum-the-odds theorem with application to a stopping game of Sakaguchi. Preprint.

Frank, A. Q. And Samuels, S. M. (1980). On an optimal stopping problem of Gusein-Zade. Stoch. Process. Appl. 10, 299-311.

Gilbert, J. P. And Mosteller, F. (1966). Recognizing the maximum of a sequence. J. Amer. Statist. Assoc. 61, $35-73$.

Gnedin, A. V. (1996). On the full information best-choice problem. J. Appl. Prob. 33, 678-687.

Gnedin, A. V. (2004). Best choice from the planar Poisson process. Stoch. Process. Appl. 111, 317-354.

GuSEIN-ZADE, S. M. (1966). The problem of choice and the optimal stopping rule for a sequence of independent trials. Theory Prob. Appl. 11, 472-476.

Hill, T. P. AND KRengel, U. (1992). A prophet inequality related to the secretary problem. In Strategies for Sequential Search and Selection in Real Time (Amherst, MA, 1990; Contemp. Math. 125), American Mathematical Society, Providence, RI, pp. 209-215.

Hsiau, S.-R. AND YANG, J.-R. (2000). A natural variation of the standard secretary problem. Statistica Sinica 10, 639-646.

Hsiau, S.-R. And YAng, J.-R. (2002). Selecting the last success in Markov-dependent trials. J. Appl. Prob. 39, $271-281$.

KnUth, D. E. (1992). Two notes on notation. Amer. Math. Monthly 99, 403-422.

Krieger, A. M. AND SAmuel-Cahn, H. (2009). The secretary problem of minimizing the expected rank: a simple suboptimal approach with generalizations. Adv. Appl. Prob. 41, 1041-1058.

Lindley, D. V. (1961). Dynamic programming and decision theory. Appl. Statist. 10, 39-51.

Mucci, A. G. (1973). On a class of secretary problems. Ann. Prob. 1, 417-427.

Presman, È. L. And Sonin, I. M. (1972). The best choice problem for a random number of objects. Theory Prob. Appl. 17, 657-668.

Samuels, S. M. (1991). Secretary problem. In Handbook of Sequential Analysis, eds B. K. Ghosh and P. K. Sen, Dekker, New York, pp. 381-405.

Samuels, S. M. (2004). Why do these quite different best-choice problems have the same solutions? Adv. Appl. Prob. 36, 398-416.

Tamaki, M. (2009). Optimal choice of the best available applicant in full-information models. J. Appl. Prob. 46, 1086-1099. 